\title{
INTEGRATING DISCRETE-EVENT AND TIME-BASED MODELS WITH OPTIMIZATION FOR RESOURCE ALLOCATION
}

\author{
Teresa Hubscher-Younger \\ Pieter J. Mosterman \\ Seth DeLand \\ Omar Orqueda \\ Doug Eastman \\ MathWorks \\ 3 Apple Hill Dr. \\ Natick, MA 01760, USA
}

\begin{abstract}
Optimization's importance for technical systems' performance can hardly be overstated. Even small improvements can result in substantial cost, resources and time savings. A constructive approach to dynamic system optimization can formalize the optimization problem in a mathematical sense. The complexity of modern systems, however, often prohibits such formalization, especially when different modeling paradigms interact. Phenomena, such as parasitic effects, present additional complexity. This work employs a generative approach to optimization, where computational simulation of the problem space is combined with a computational optimization approach in the solution space. To address the multi-paradigm nature, simulation relies on a unifying semantic domain in the form of an abstract execution framework that can be made concrete. Because of the flexibility of the computational infrastructure, a highly configurable integrated environment is made available to the optimization expert. The overall approach is illustrated with a resource allocation problem, which combines continuous-time, discrete-event, and state-transition systems.
\end{abstract}

\section{INTRODUCTION}

The increasing exploitation of automation in technical systems combined with their consistently growing scale has rendered optimization of the operation of such systems an ever more valuable exercise. For example, with day rates as high as $\$ 100,000$ and over, a small improvement of the trajectory that an ultra large crude carrier tanker sails comes with significant returns (Arnsdorf 2012, Nightingale 2010). As another example, reducing the carbon monoxide produced by a single vehicle has a multiplier effect that may be of the order of millions of cars sold (e.g., in 2008 alone Toyota sold as many as 351,007 Toyota Corolla (Toyota 2009)). As a consequence even small performance improvements are likely to have substantial impact in terms of cost, resources used, time spent, etc. Thus, optimization plays a role of key importance in the design of technical systems across the entire spectrum of industry and application domains.

To formally approach the optimization problem for technical systems, it is useful to formulate the problem in a mathematical sense, thereby opening up the opportunity to exploit the extensive body of mathematics literature on optimization. Indeed, once a mathematical formulation is available, optimization methods abound, including calculus-based methods, linear programming, integer programming, dynamic programming, calculus of variations, etc. (Nemhauser and Wolsey 1999, Rao 2009). Such optimi- 


\section{Hubscher-Younger, Mosterman, DeLand, Orqueda, and Eastman}

zation often requires formulating the problem according to a mathematical structure that is then amenable to be combined with a mathematical solution approach.

While such an approach of optimization by construction often achieves excellent results, it involves a complicated and intricate step where the optimization of a technical system in a given sense must be transformed into a formulation amenable to the mathematical solution approach. This can prove to be a challenging task, in particular if the technical system is modeled such that it embodies a variety of models of computation. Moreover, many times the task is further complicated because the optimization is over properties that make it necessary to account for critical parameters that correspond to detailed phenomena. Such detailed phenomena are especially difficult to handle in a canonical mathematical formulation when they are difficult to abstract, for example because their effect is crucial in the combination of various different models of computation. Concretely, effects such as parasitic resistances and capacitances may be essential in properly modeling a system as a combination of continuous-time and discrete-event model parts. Yet such effects are likely to (prohibitively) complicate the optimization problem.

In addition to the combination of various models of computation in the problem space, the solution approach contributes its own model of computation, time scales, etc. In other words, the optimization approach itself often operates in a different model of computation than the problem space. For example, while a manufacturing operation may operate on a daily time scale, the optimization of the process may take a weekly or even monthly time scale and a model of computation different from the problem space may be chosen to (i) represent the solution approach and (ii) efficiently support the time scale of the solution approach.

With the different time scales between the optimization and the problem under optimization naturally comes the desideratum to change the problem under optimization over the course of operation and through various cycles of optimization. Facilitating dynamic problem adaptation necessitates automatically generating a new optimization problem, for example from an original model. However, while achievable in restricted cases, a more general such automation approach is a challenging undertaking.

Instead of a constructive approach the work presented in this paper employs a generative approach to mitigate many of the issues. As opposed to incorporating deep knowledge of the optimization problem in a formalization such that it can be exploited by the solution approach, a flexible and powerful interface between the problem and the solution space is defined. This interface enables the use of sophisticated simulation algorithms on the problem side and a broad spectrum of optimization methodologies on the solution side. Previous work based on a Knowledge Interface Broker (KIB) has demonstrated the value of such a generative approach where discrete event simulation is combined with Modeling Predictive Control (MPC) optimization algorithms that operate on a much larger time scale (Godding, Sarjoughian and Kempf 2007). While the knowledge interface broker introduces an elegant concept in solving the necessity for semantic adaptation between the problem and solution space, this paper presents a refinement based on an integrated architecture that eliminates the complexity of combining disjoint tools.

A main benefit of an integrated architecture is that it eliminates the complexity of having different tools with their various idiosyncratic nuances in the syntax, static and dynamic semantics of both interface as well as behavior. The challenge it poses in return is that it still requires the integration of problem and solution formulations, where these formulations often rely on different formalisms with different syntax and different semantics and where the problem and solution may operate on widely differing temporal scales. Moreover, within the problem formulation various different formalisms may be employed and so the overall challenge takes on a distinct multi-paradigm modeling character (Mosterman and Vangheluwe 2004).

This work addresses the multi-paradigm modeling challenge for optimization by developing an integrated optimization architecture with the objective of providing a unifying semantic domain (Harel and Rumpe 2000). Based on a well-defined abstract model of computation, elements in the architecture can register specific concrete refinements to implement various models of computation. An overall architecture framework around the abstract model of computation facilitates the integration of the various 


\section{Hubscher-Younger, Mosterman, DeLand, Orqueda, and Eastman}

refinements and provides a coherent and homogeneous optimization environment for multi-paradigm problems. As a case study, an optimization problem is formulated as model-predictive control of a batch process. Where the problem includes discrete-event and continuous-time models of computation, the solution approach relies on a control flow model of computation. The developed architecture then provides an integrated environment that enables direct experimentation with the different parts of the problem as well as the solution approach without changing development environments or refactoring interface brokers.

Section 2 of this paper first provides background to integrated optimization approach that is presented. Section 3 then discusses integration of different modeling paradigms. Section 4 introduces the case study model to be optimized, a chemical batch production model. Section 5 describes the optimization approach using MATLABß.

\section{BACKGROUND}

The need for information aggregation and separation of input and output data for different modeling and optimization paradigms, based on syntactic and semantic simulation, as well as different time scales has proven to be a complex problem. The KIB approach with its specification of a set of nodes to handle the passing of information to the Discrete Event Simulation and Model Predictive Controller is useful for its ability to act as a transmitting and receiving layer for the knowledge that needs integration between the environments (Huang et al. 2009). The KIB provides a communication layer that allows the designers of the two different environments to act independently, but the integration is difficult to do during model generation.

$\mathrm{As} \mathrm{Fu} \mathrm{(2002)} \mathrm{argues,} \mathrm{optimization} \mathrm{in} \mathrm{the} \mathrm{solution} \mathrm{space} \mathrm{and} \mathrm{discrete-event} \mathrm{simulation} \mathrm{in} \mathrm{the} \mathrm{problem}$ space should be more integrated to take advantage of the fact that the two tasks are performed together. However, he also argues that integration of optimization into discrete-event simulation software can take a bad turn, when the user is supplied GUIs that hide the specific algorithms used and prevent the user from customizing the optimization routine. April, Glover, Kelly and Laguna (2003) argue a similar point when they describe the "metaheuristic black-box approach" in commercial software implementations of simulation optimization. Approaches often do not work well out-of-the-box, but need to be adapted to the specific characteristics of a problem.

The flexibility in MATLAB® (2012) and openness of its programming environment to optimization algorithms allows both integration with the simulation and the control for customization that is also desired for optimization routines that go beyond simple rules provided by the simulation tool.

Others have expressed the need for knowledge transfer between Model Predictive Control (Qin and Badgwell 2003) at one level of abstraction and Discrete Event System Specification (Zeigler and Sarjoughian 2000) at another. The challenge is that we must deal with the information exchange between enterprise system, which are consists of a base system, operative system, control and monitoring system and planning system (Mönch, Lendermann, McGinnis, and Shirrmann 2011).

We address some of these challenges in MATLAB through three types of integration:

1. Integration of multi-domain models using gateways

2. Integration through a base mathematical programming language

3. Parallel simulation of the hybrid model

\section{INTEGRATION OF MULTI-DOMAIN MODELS}

Integrating various modeling paradigms into a highly configurable and flexible environment requires support for combining continuous and discrete behavior, both in space and time. Such support comprises not only the ability for a user to express a notion in a particular paradigm but also the facilities for efficient computational algorithms operating on the paradigms. For example, a continuous-time model may be specified as a differential equation which is often solved computationally by applying numerical integration algorithms in a time stepping fashion (Mosterman, 2007). In contrast, a discrete-event model 


\section{Hubscher-Younger, Mosterman, DeLand, Orqueda, and Eastman}

may be specified as a discrete event system specification (DEVS) (Zeigler 1976) which is often solved by an event-driven approach, for example based on an event calendar.

In this work, continuous-time models are specified as time-based block diagrams, which are supported by the Simulink ${ }^{\circledR}$ (2012) graphical modeling environment. In addition to continuous-time, these timebased block diagrams support both discrete time with the notion of a sample rate as a first-class citizen of the modeling language. Discrete-event models are either specified as state transition diagrams, supported by the Stateflow ${ }^{\circledR}$ (2012) graphical modeling environment, or as entity flow diagrams, supported by the SimEvents ${ }^{\circledR}$ (2012) graphical modeling environment. In addition, untimed discrete event models are supported by the control flow approach of the MATLAB technical computing environment.

An integrated framework that supports all these various environments and their combinations is based on the S-Function Application Programming Interface (API) in Simulink. This API provides an abstract structure of a set of foundational execution operations. A subset that is of interest in the scope of this paper comprises

- An output operation to evaluate a combinational (Sander and Jantsch 2004) computation (i.e., a functional computation without side effects such as modifying state).

- An update operation to evaluate a sequential (Sander and Jantsch 2004) computation (i.e., a computation that modifies the state of a dynamic system).

- A derivatives operation to obtain the derivative with respect to time of a model variable.

- A function-call operation to explicitly invoke an output operation, possibly combined with an update operation.

Function-calls are used with a type of subsystem or block that is invoked as a function by another block. In other words the function-call is a trigger that causes action in the component that the function call signal connects to.

An execution engine based on this framework then provides support for solving differential equations (implemented by making the output and derivative operations concrete), difference equations (implemented by making the output and update operations concrete), state transition behavior (implemented by making the function-call operations concrete), and entity flow behavior (implemented by making the function-call operations concrete and embedding an event calendar). Because of the shared framework, Simulink, Stateflow, and SimEvents modeling environments can be integrated to design models with the semantic heterogeneity that is often desired for complex optimization problems. Simulink and SimEvents allow discrete-event and time-based simulation models to be integrated with one another. The SimEvents engine works in conjunction with the different Simulink solvers to allow multi-domain modeling of complex systems.

In terms of model language elements, semantic clarity and precision is paramount. In this context, it is of particular importance to define the semantics of the interface between modeling paradigms (Hardebolle and Boulanger 2007). In this work, because of the integrated framework, the critical interface is between the imperative event-based entity flow diagrams of SimEvents and the declarative time-based block diagrams of Simulink. To precisely define this interface, the imperative control that explicitly invokes an operation must be reconciled with a declarative formulation that is devoid of control over when an operation is invoked. A set of gateway blocks provide the user with language elements to specify the semantic adaptation. Signals coming out of or driving the SimEvents model can be used in the time-based domain of the Simulink model via a component called the Gateway block. These Gateway blocks do signal conversion for use in the different domains. There are four types of Gateway blocks in SimEvents shown in Figure 1.

- Timed to Event Signal and Event to Timed Signal blocks adapt the event-driven imperative and the time-driven declarative environments of SimEvents and Simulink, respectively. The output signal of the Timed to Event Signal gateway block is identical to that of the input signal, except that now this signal can be used as an event-based input signal. The output signal of the Event to Timed Signal gateway block can be used for modeling time-based dynamics, and thus gives one value at any given time on the simulation clock. The output signal has a 
sample time type of "fixed in minor step", in order to have sample time hits that are synchronized to the other time-based signals in the model.

- Timed to Event Function-Call and Timed to Event Function-Call blocks adapt the imperative state transition diagrams of Stateflow (with time derived from the overall execution engine) and the imperative entity flow diagrams of SimEvents (with time derived from the embedded event calendar). The Event to Timed Function-Call block converts the function-call from the discrete-event domain into one that can be used in a time-based domain, whereas the Timed to Event Function-Call converts the signal in the other direction.

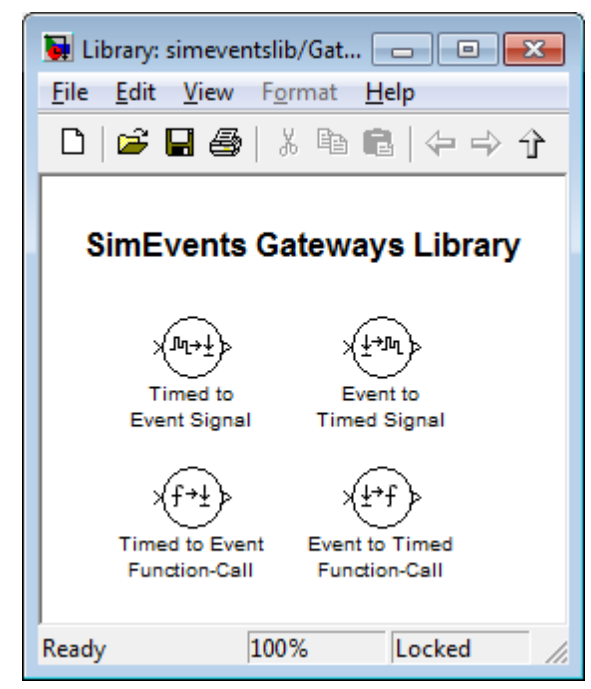

Figure 1: Gateway blocks that are part of the SimEvents Gateways Library help transform signals from a discrete-event system to work in a time-based system and vice versa.

These gateway blocks clearly define where system boundaries and delineation of the two types of systems are, as well as allows for the passing of information from one type of system to another. We will demonstrate the use of these blocks in the example that follows.

\section{THE PROBLEM TO BE OPTIMIZED-CHEMICAL BATCH PRODUCTION}

The model the authors created to demonstrate how we support integration is a hybrid, discrete-event and time-based model that simulates a chemical batch production process with shared resources that processes two types of orders. There are three main components to this model (Figure 2): the order system, production system and process analysis. The order system generates orders with different demands on the production resources. The production system the loop in the middle, is assigning batch reactors to a particular order and processing this order. The process analysis component displays how the chemical production process resources were used over time. 


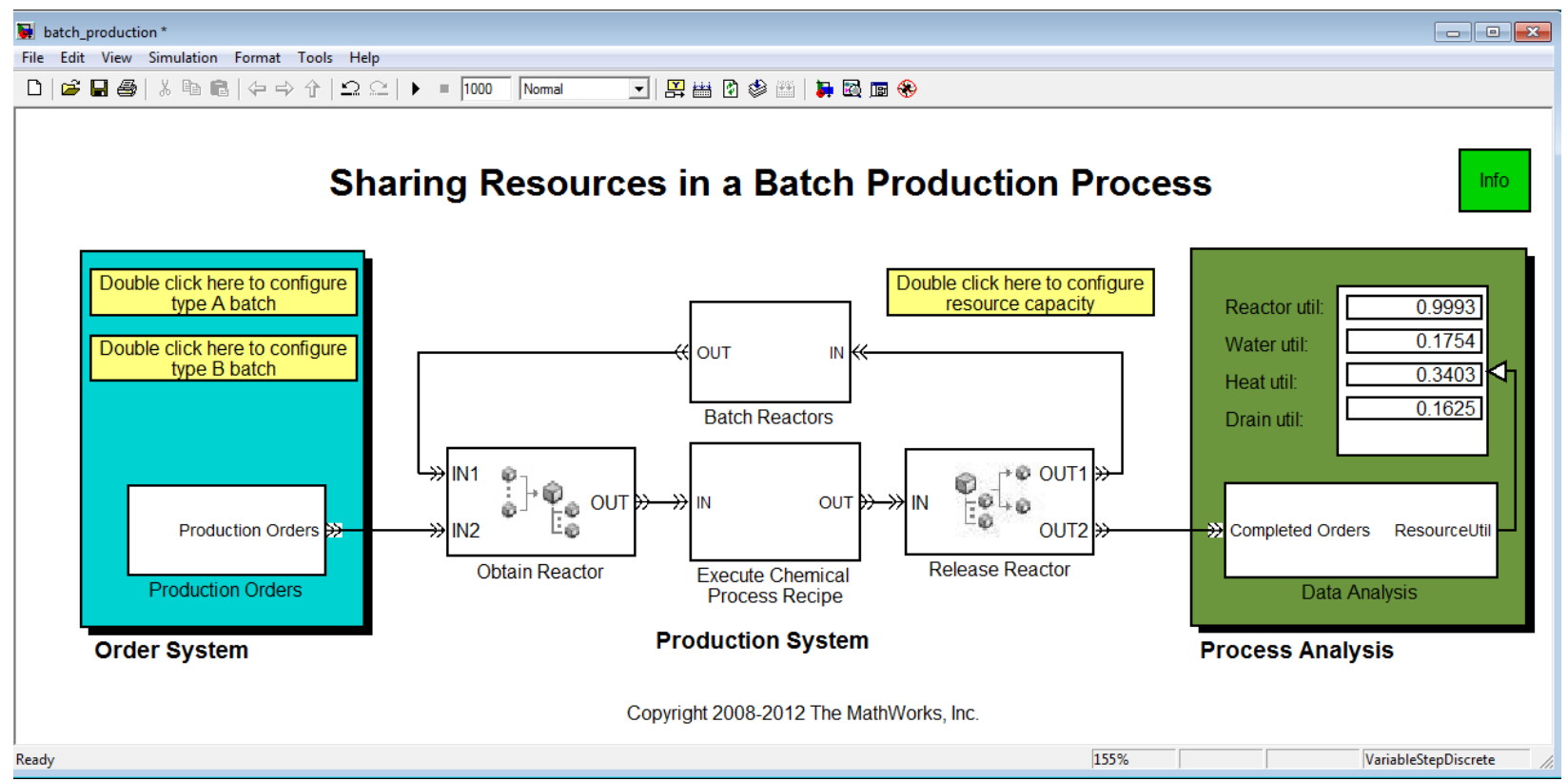

Figure 2: The chemical batch production model has three main components at the top level: the order system, the production system and the process analysis component.

The Production Orders subsystem (Figure 3) simulates the generation and backlog of two types of production orders, an $\mathrm{A}$ and $\mathrm{B}$ batch and then holds the order in a queue until a reactor is ready. A situation where this might be applicable is where the A batch being a weaker strength of a particular pharmaceutical and B being a stronger one. They each would require different amounts of time of the shared resources to be available. 


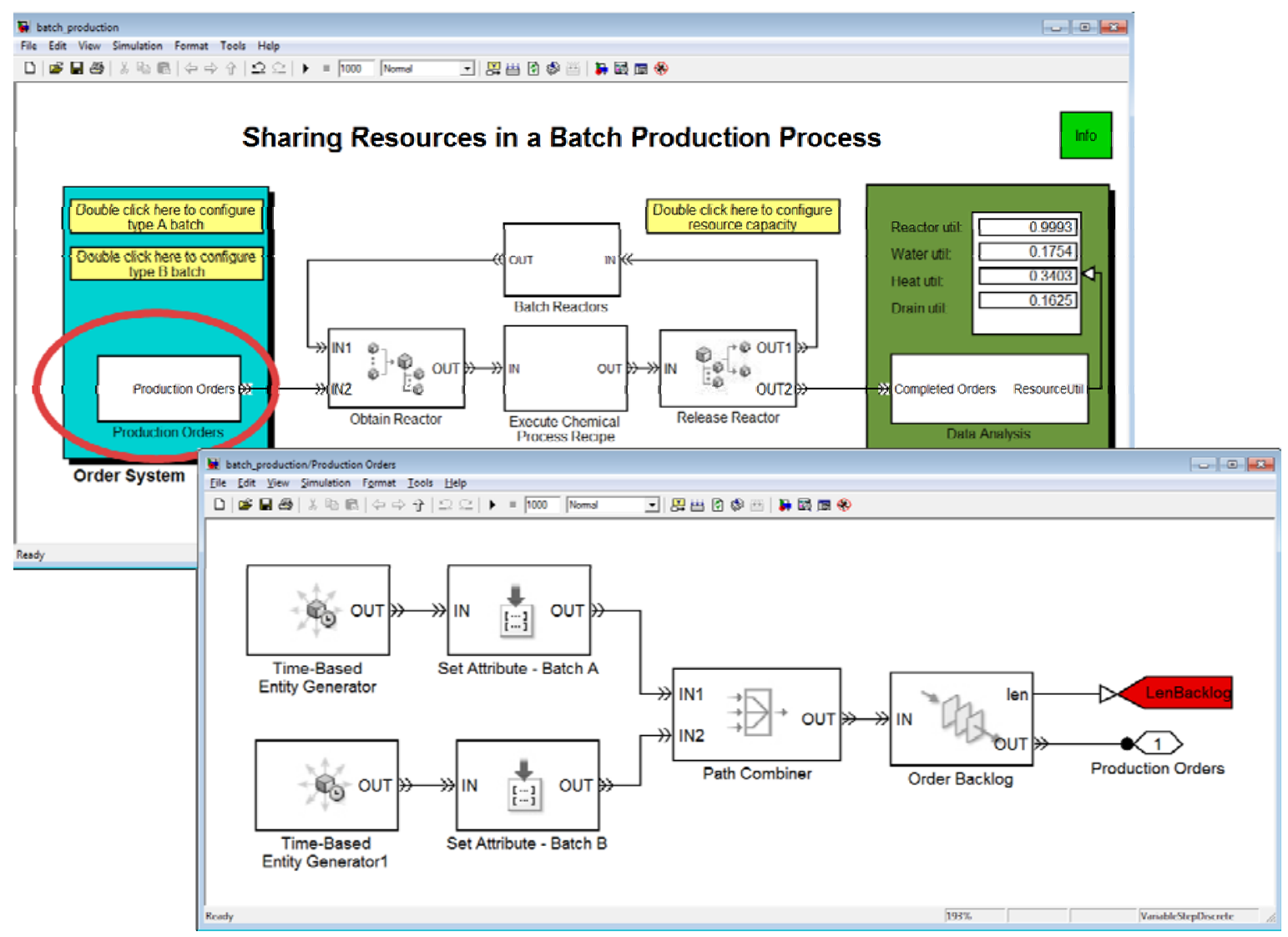

Figure 3: The discrete-event subsystem of the Production Orders component that creates two different batches of orders.

The Batch Reactors subsystem (Figure 4) models batch reactors, one of the main shared resources in this production process. One reactor is used to process one production order or batch. The Time-Based Function-Call Generator and Event-Based Entity Generator generate the batch reactors at the beginning of the simulation. The FIFO Queue block holds the reactors until they are needed. The Path Combiner block adds back into the process batch reactors that have been used and are ready to be used for another order. 


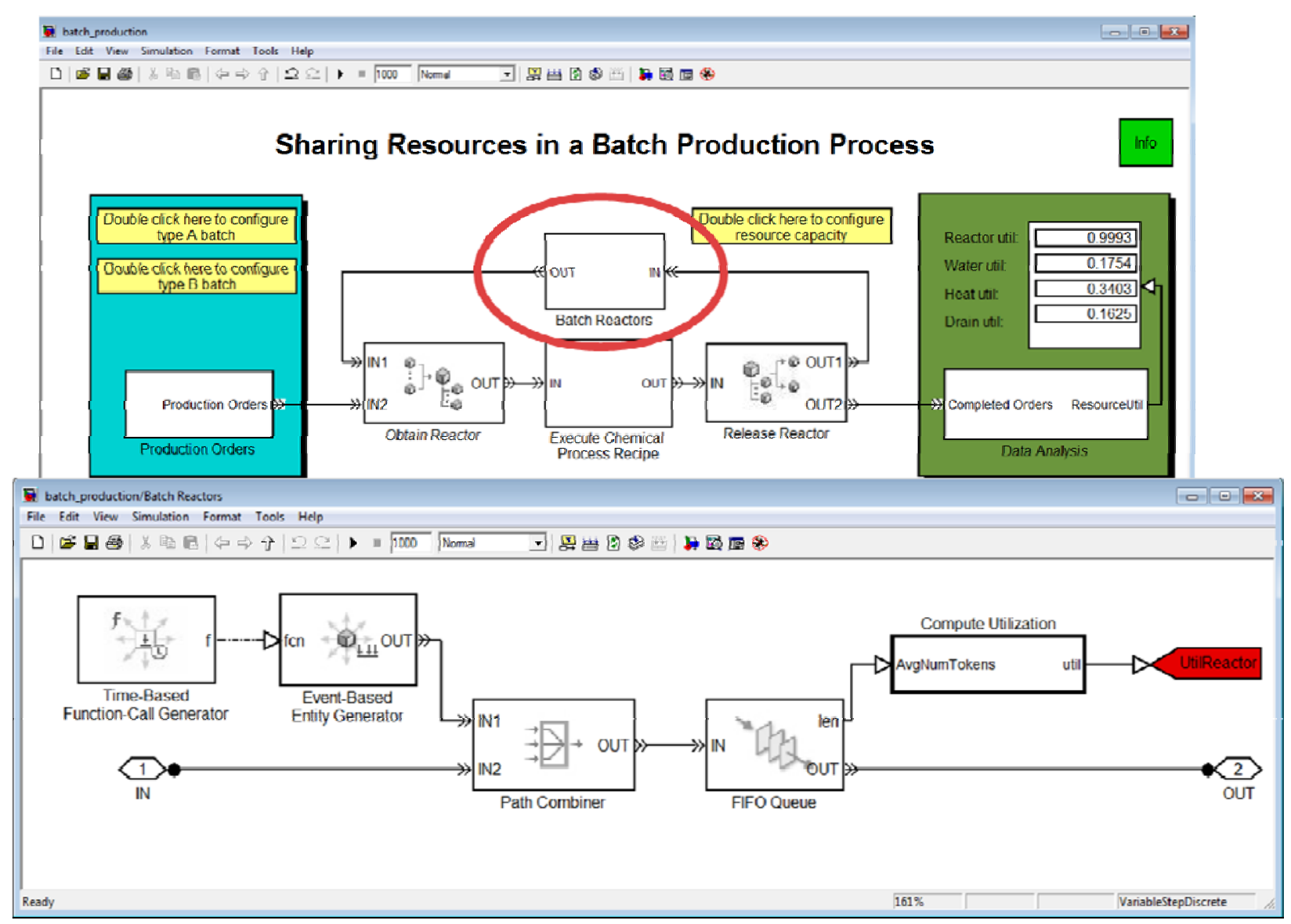

Figure 4: The Batch Reactors subsystem creates batch reactors at the beginning of the process, which are reused during simulation.

In the main model, once a particular production order is received, the block labeled Obtain Reactor requests a reactor from the set of batch reactors held in the Batch Reactors subsystem. This begins the chemical process. Inside the Chemical Process Recipe, there are a number of manufacturing steps, such as adding water, heating the mixture, adding color and particles, agitation and draining. (Figure 5). Once the Chemical Process Recipe is complete, then the batch reactor is released to be used for another order. 


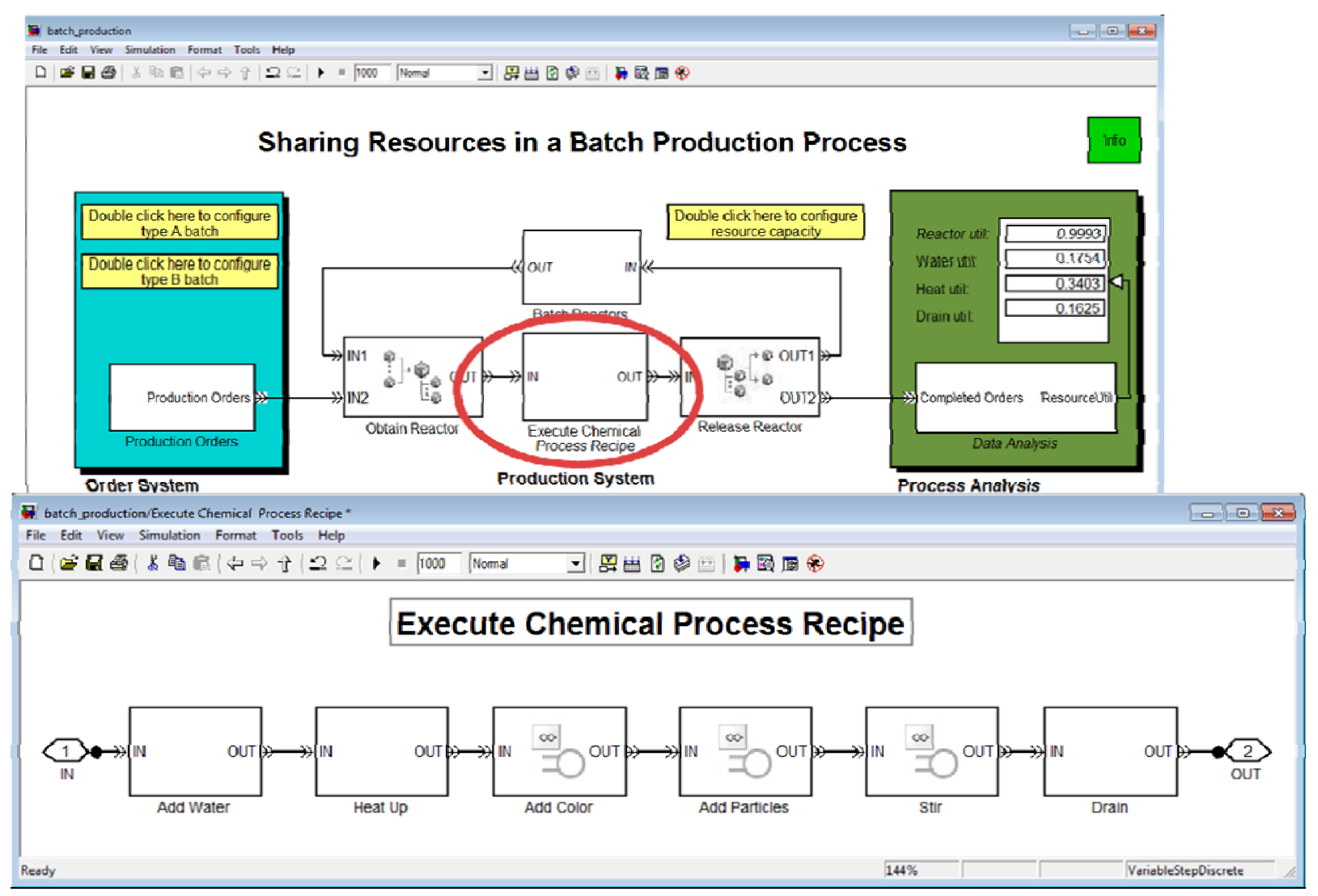

Figure 5: The Execute Chemical Process Recipe subsystem has the major steps of the batch reactor.

One of the subsystems, the Heat Up system, has a continuous time controller in the loop. This is where we see the usefulness of the gateway blocks. The Chemical Reactor Controller is a subsystem containing a continuous time MPC controller inside it, so it has two gateway blocks. One on the signal coming out of the discrete-event system which sets the feed concentration, and another gateway block that is used for enabling further orders from moving through the system based on the state of the controller (Figure 6). 


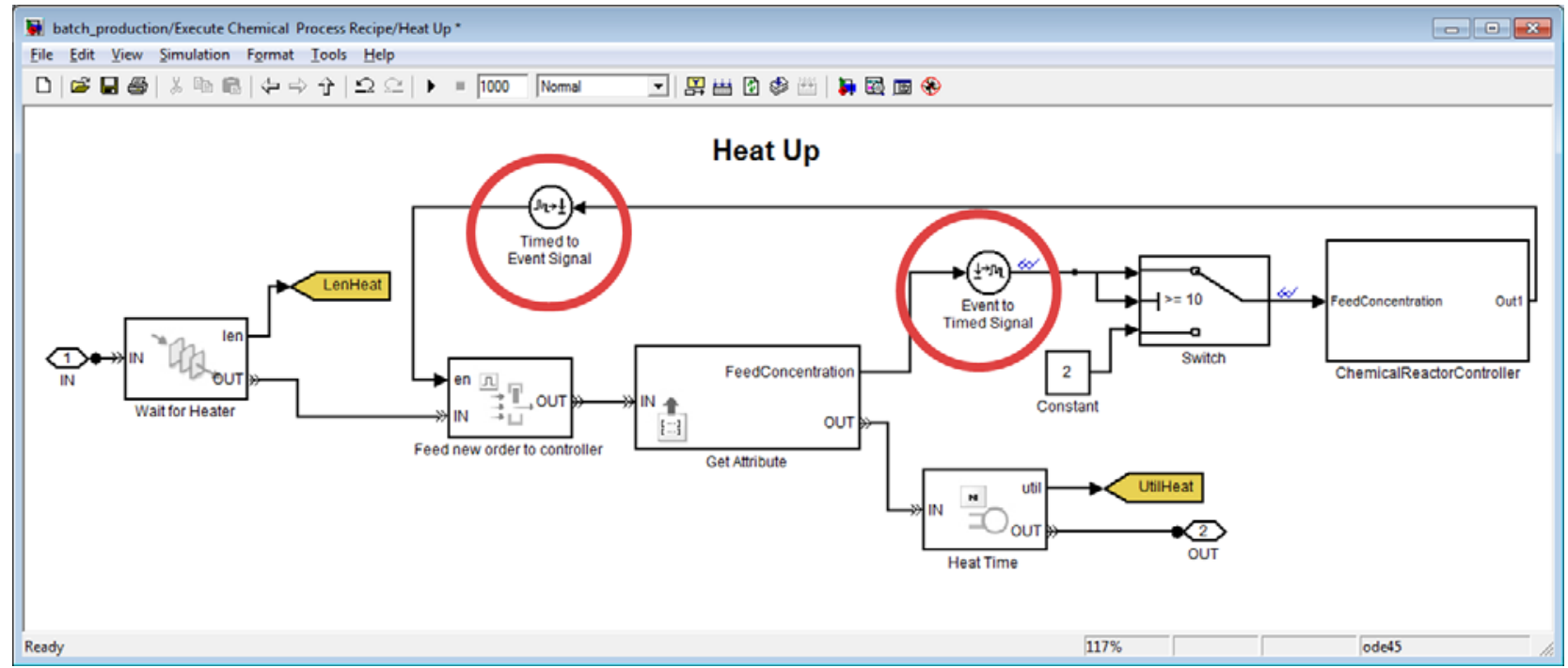

Figure 6: At the interfaces to the subsystem containing the continuous time controller, there are gateway blocks to convert signals from the event-based and time-based domains.

Inside the subsystem, the controller has a MPC continuous time controller, a plant model and a Stateflow chart, that allows us to use state logic to send a signal to block orders based on the state of the controller (Figure 7). Hybrid modeling in Simulink allows integration of controller, plant and discreteevent system.

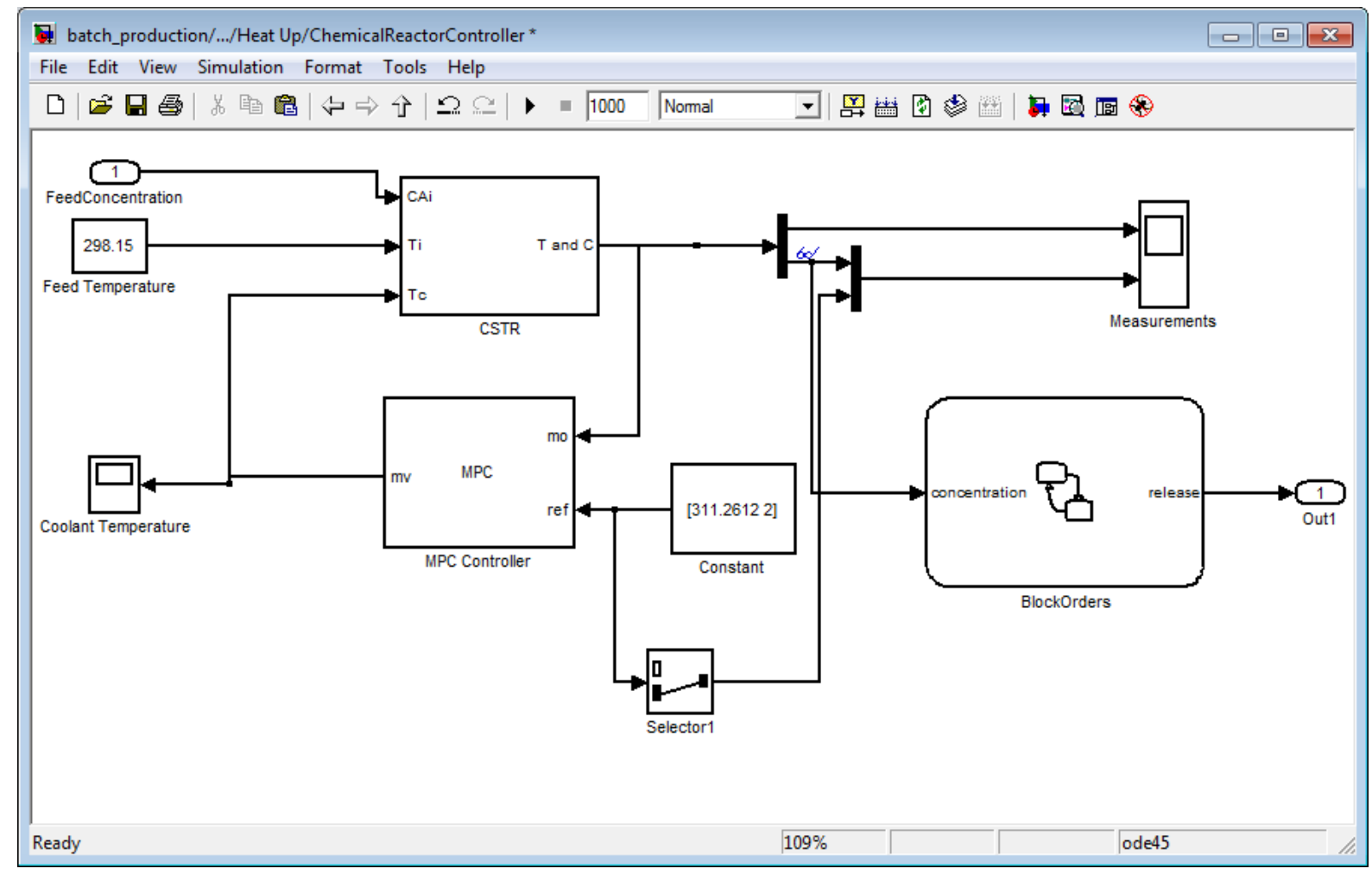

Figure 7: This subsystem within the discrete-event model contains a continuous time controller, plant, and state logic that blocks orders when the concentration is too high. 
Upon simulation of this model, one can inspect different simulation output results and system characteristics, including the utilization of individual resources, average order backlog, or throughput.

\section{THE OPTIMIZATION APPROACH}

The MATLAB software environment offers a computational infrastructure for optimizing hybrid, discrete-event and time-based, models, which allows for a great deal of flexibility in scripting and modifying the optimization objective function, while also making it easier to tie together parallel discrete simulation and optimization without the pain of the context switching into multiple software environments.

To demonstrate how the tools can be used together effectively and efficiently, we will use a genetic algorithm from the Global Optimization Toolbox (2012) to find a suitable combination of equipment we need to purchase (batch reactors, water tanks, heaters and drains) to minimize the backlog of orders while taking into account the cost of adding resources to the process. The reason we choose a genetic algorithm is because it supports mixed integer optimization, important for this example, as the number of resources are integers, rather than continuous values, thus making the problem harder to solve.

\subsection{Optimization Script}

The genetic algorithm will minimize a customized objective function programmed in MATLAB. The first section in the objective function sets the values for the number of batch reactors, water tanks, heaters and drains in the model. The function set_param lets you set parameter values in Simulink from MATLAB.

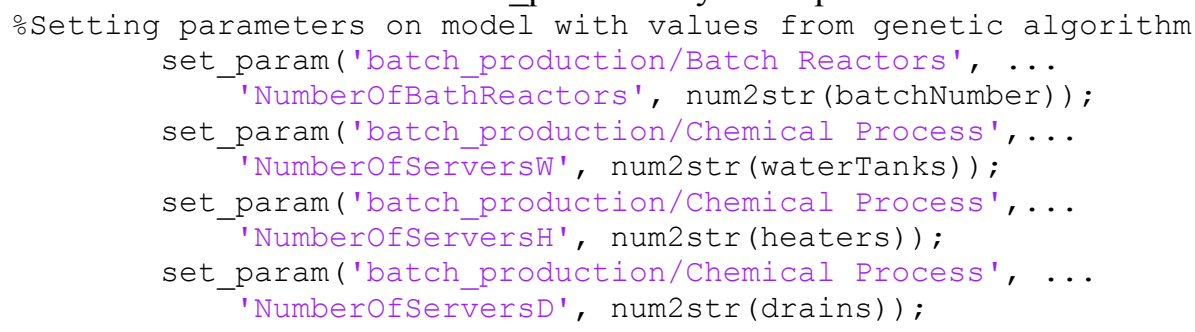

Then the objective function simulates the model by using the sim function, and captures the order backlog in the output variable called backlogValues.

oSimulation and capturing the output

simout = sim('batch_production', 'Saveoutput', 'on',...

'OutputSaveName',' 'backlogValues') ;

The objective function is then calculated as a function of the backlog and the cost of adding more resources. The algorithm will run the simulation multiple times with the objective of minimizing both the backlog and the cost of adding in more resources.

\subsection{A Parallel Implementation}

Optimization, decision making, and data aggregation systems often need to execute multiple simulations. Parallel simulations speed up these systems and algorithms considerably. The Parallel Computing Toolbox (2012) supports solving problems and running multiple simulations using multicore processors, GPUs and when coupled with the MATLAB ${ }^{\circledR}$ Distributed Computing Server ${ }^{\mathrm{TM}}$ (2012) on computer clusters.

Parallel for-loops, special array types and parallelized numerical algorithms, such as the genetic algorithm allows one to parallelize MATLAB applications without using programming languages outside of MATLAB. Parallel Computing Toolbox ${ }^{\mathrm{TM}}$ is used with Simulink to run multiple simulation of a model in parallel. The toolbox provides 12 workers to execute applications locally on a multicore desktop, and without changing the code, one can run the same application on a computer cluster. 
The optimization script for the problem below shows how parallel simulation is built into the genetic algorithm we used from the Global Optimization Toolbox and therefore makes simulation on multiple cores easier to set-up and run.

In MATLAB, one can create or import cluster profiles for parallel runs. These profiles define the cluster of workers that can work on a job in parallel, load in files that one needs to do the job and store the data in the space one specifies. To access the profile, one types the name of the pool profile with the number of workers to use. For this example, the profile is named "speedy" with 16 workers being used in parallel on a cluster.

>> matlabpool speedy 16

The next step is to load the model onto all the workers being used.

>> pctRunOnAll ('load_system(' 'batch_production' ')')

The next step is running the script for optimization we have defined above.

>> resources $=$ optimizationBatchProcessParallel()

As the simulation runs, two plots appear that were specified in the optimization options (Figure 8). The first plot shows the penalty values, as well as the best penalty value it has found and the mean value. The second plot shows the current best values for the different resources. In our case, the first value is the suitable number of batch reactors, the second is the number of water tanks, third is the number of heaters, and the fourth number is the suitable number of drains to use.

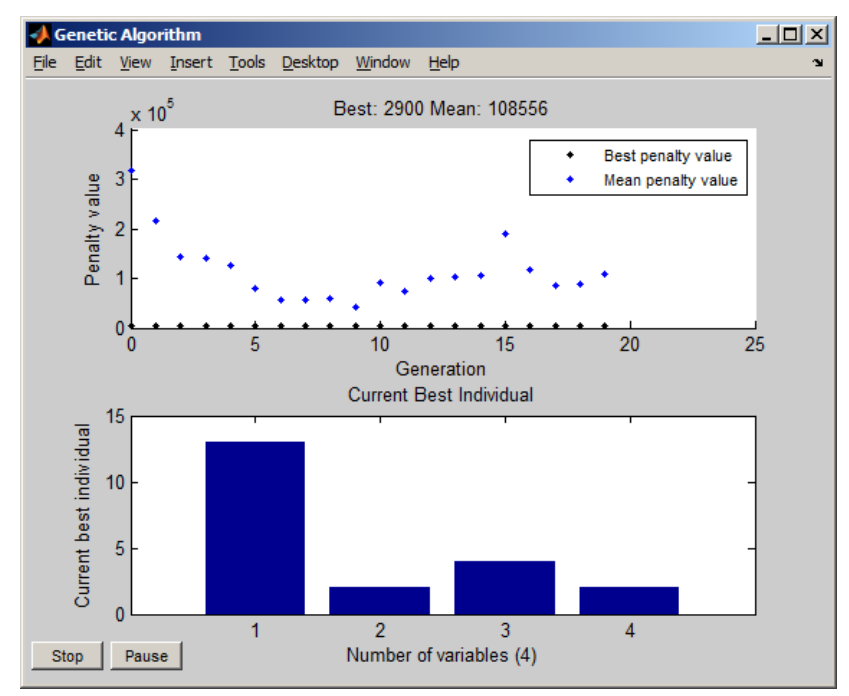

Figure 8: The plots chosen for the genetic algorithm show the progress of the algorithm as well as the best individual values found by the genetic algorithm for the resources we wish to optimize.

\subsection{Experimental Results}

The results in MATLAB (Figure 9) show that the process took 175 seconds and that it found the suitable numbers of resources to be 13 batch reactors, two water tanks, four heaters and two drains. The SimEvents model ran 400 times (population of 20x20 generations). There are 2,500 possible configurations for this model, but the genetic algorithm found a suitable one after only trying 400 . 


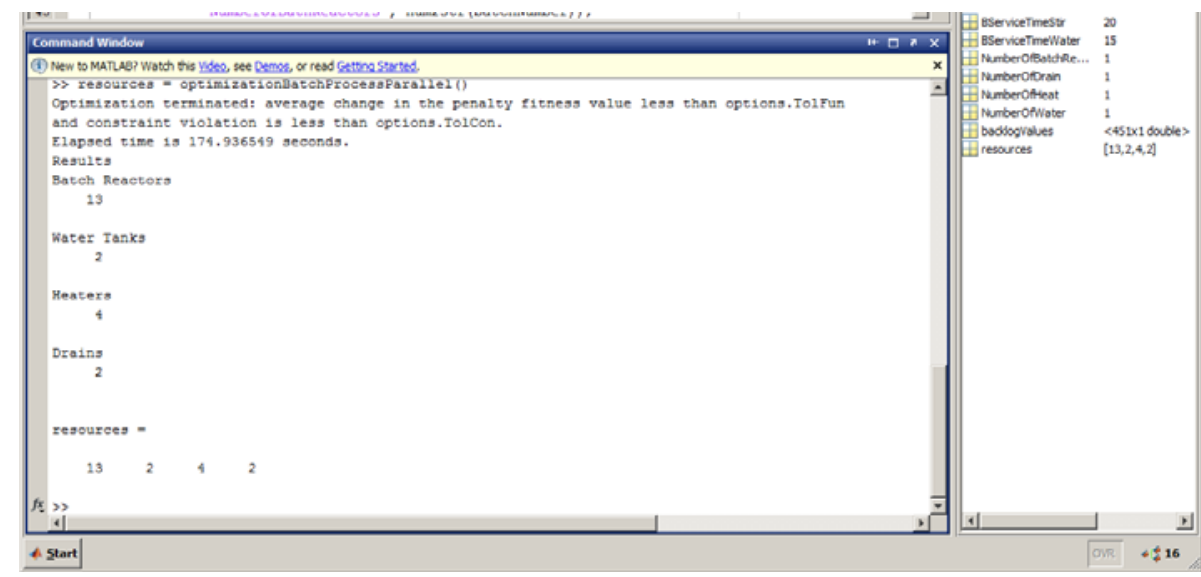

Figure 9: The optimization took 175 seconds and shows the number of the different resources needed.

\section{CONCLUSIONS}

This work demonstrates how the authors support a generative approach to optimization, where computational simulation is combined with computation optimization. Various models of computation can be implemented and combined using a unifying semantic domain in the form of an abstract execution framework. Integrating continuous and discrete behaviors through gateway blocks, using MATLAB as a unifying mathematical programming environment and parallel simulation tools help to make this a pragmatic approach for optimization specialists. As a case study, a chemical batch production process was optimized using its integrated approach.

The next step for our model and research is to take it in the direction that Venkateswaran, Son, and Jones (2004) proposed where we integrate an Enterprise-level production planner and a system dynamics model of the enterprise that feeds into the model actual inventory levels and orders, rather than using distributions of predefined sets of orders. Expanding the model to capture more of the workflow of building in the simulation in bigger decision-making systems and therefore integrating them with Business Information Systems is a challenge described by Mönch and colleagues (2011), that we believe is worth further pursuit.

\section{REFERENCES}

April, J., Glover, F., Kelly, J.P., \& Laguna, M. 2003. "Practical Introduction to Simulation Optimization." Proceedings of the 2003 Winter Simulation Conference, pp. 71-80.

Arnsdorf, I. 2012. "OPEC Pledge Means Record Tanker Cargoes Reversing Rout: Freight." Bloomberg News, June 20, 2012. Retrieved June 2012 from: http://www.bloomberg.com/news/2012-06-19/opecpledge-means-record-tanker-cargoes-reversing-rout-freight.html.

Fu, M.C. 2002. "Optimization for Simulation: Theory vs. Practice.” INFORMS Journal on Computing, pp. 192-215.

Global Optimization Toolbox 2012, March. Global Optimization Toolbox User's Guide. Natick, MA: The MathWorks.

Godding, G. Sarjoughian, H., Kempf, K. 2003. "Semiconductor supply network simulation." Proceedings of the 2003 Winter Simulation Conference, pp. 1593-1601.

Godding, G., Sarjoughian, H., \& Kempf, K. 2007. "Application of Combined Discrete-Event Simulation and Optimization Models in Semiconductor Enterprise Manufacturing Systems." Proceedings of the 2007 Winter Simulation Conference, pp. 1729-1736. 
Hardebolle, C. and Boulanger, F. 2007, October. "ModHel'X: A Component-Oriented Approach to MultiFormalism Modeling." Proceedings of the 2007 Workshop on Multi-Paradigm Modeling (MPM'07) at the 10th IEEE/ACM International Conference on Model-Driven Engineering Languages and Systems (MODELS 2007). BME-DAAI Technical Report Series. Nashville TN, United States. p. 49-60.

Huang, D., Sarjoughian, H.S., Wang, W., Godding, G., Rivera, D.E., Kempf, K. G., \& Mittelmann, H. 2009. "Simulation of Semiconductor Manufacturing Supply-Chain Systems with DEVS, MPC, and KIB." IEEE Transactions on Semiconductor Manufacturing, 22 (1).

MATLAB 2012, March. MATLAB ${ }^{\circledR}$ Primer. Natick, MA: MathWorks.

MATLAB Distributed Computing Server 2012, March. MATLAB ${ }^{\circledR}$ Distributed Computing Server User's Guide. Natick, MA: MathWorks.

Mönch, L., Lendermann P., McGinnis, L. F., \& Shirrmann, A. 2011. “A survey of challenges in modelling and decision-making for discrete event logistics systems." Computers in Industry, 62, pp. 557567.

Mosterman, P.J. 2007. "Hybrid Dynamic Systems: Modeling and Execution." Handbook of Dynamic System Modeling, Paul A. Fishwick (editor), Chapter 15, CRC Press.

Mosterman, P. J. \& Vangheluwe, H. 2004, September. "Computer Automated Multi-Paradigm Modeling: An Introduction." SIMULATION: Transactions of The Society for Modeling and Simulation International, 80 (9), pp. 433-450.

Nemhauser, G. L. \& Wolsey, L. A. 1999. Integer and Combinatorial Optimization. Wiley Interscience, New York. ISBN 0-47-135943-2.

Nightingale, A. 2010. "Supertankers Face Losing Run as Frontline Shuns Oil.” Bloomberg News, Oct. 25, 2010. Retrieved June 20, 2012 from: http://www.bloomberg.com/news/2010-10-24/supertankersface-longest-losing-streak-in-17-years-as-frontline-shuns-oil.html.

Parallel Computing Toolbox 2012, March. Parallel Computing Toolbox User's Guide. Natick, MA: The MathWorks.

Qin, S. J, \& Badgwell, T. A. 2003. "A survey of industrial model predictive control technology." Control Engineering Practice, pp. 369-393.

Rao, S. 2009. Engineering Optimization: Theory and Practice. John Wiley \& Sons, Inc., Hoboken, NJ. ISBN 978-0-470-18352-6.

Sander, I. and Jantsch, A. 2004, January. "System modeling and transformational design refinement in ForSyDe." IEEE Transactions on Computer-Aided Design of Integrated Circuits and Systems, 23(1), pp.17-32.

SimEvents 2012, March. SimEvents ${ }^{\circledR}$ User's Guide. Natick, MA: MathWorks.

Simulink 2012, March. Simulink® User's Guide. Natick, MA: MathWorks.

Stateflow 2012, March. Stateflow ${ }^{\circledR}$ User's Guide. Natick, MA: MathWorks.

Toyota 2009. "Toyota Reports 2008 and December Sales." Toyota USA Newsroom. Retrieved May 24, 2012 from: http://pressroom.toyota.com/article_display.cfm?article_id=1611.

Venkateswaran, J., Son, Y.-J., Jones, A. 2004."Hierarchical production planning using a hybrid system dynamic-discrete event simulation architecture." Proceedings of the 2004 Winter Simulation Conference, 2004, pp. 1094-1102.

Zeigler, B. (1976). Theory of Modeling and Simulation. Wiley Interscience, New York. ISBN 0-12778455-1.

Zeigler, B. P. \& Sarjoughian, H.S. 2000. "Distributed simulation: creating distributed simulation using DEVS M\&S environments." Proceedings of the 2000 Winter Simulation Conference.

\section{AUTHOR BIOGRAPHIES}

TERESA HUBSCHER-YOUNGER is the Product Marketing Manager for Discrete Event Simulation at MathWorks in Natick, MA. Before, she was an Assistant Professor at Rensselaer Polytechnic University in Rochester, NY. She has a Ph.D. degree in Computer Science and Software Engineering from Au- 
burn University in Auburn, AL., and a M.Sc. degree in Human-Computer Interaction from Georgia Institute of Technology. Her email address is Teresa.Hubscher-Younger@mathworks.com.

PIETER J. MOSTERMAN is a Senior Research Scientist at MathWorks in Natick, MA and an Adjunct Professor at the School of Computer Science of McGill University. Before, he was a Research Associate at the German Aerospace Center (DLR) in Oberpfaffenhofen. He has a Ph.D. degree in Electrical and Computer Engineering from Vanderbilt University in Nashville, TN, and a M.Sc. degree in Electrical Engineering from the University of Twente, Netherlands. His primary research interests are in Computer Automated Multiparadigm Modeling with principal applications in design automation, training systems, and fault detection, isolation, and reconfiguration. Dr. Mosterman designed the Electronics Laboratory Simulator that was nominated for The Computerworld Smithsonian Award by $\mathrm{Mi}_{\text {- }}$ crosoft Corporation in 1994. In 2003, he was awarded the IMechE Donald Julius Groen Prize for his paper on the hybrid bond graph modeling and simulation environment HyBrSim. In 2009, he received the Distinguished Service Award of The Society for Modeling and Simulation International (SCS) for his services as Editor-in-Chief of SIMULATION: Transactions of SCS. His web page is http://msdl.cs.mcgill.ca/people/mosterman/. His email address is pmosterman@yahoo.com

SETH DELAND is Product Marketing Manager of Optimization at MathWorks in Natick, MA. He holds a M.S. in Mechnical Engineering from Michigan Technological University in Houghton, MI. His email address is Seth.DeLand@mathworks.com.

OMAR ORQUEDA is a Software Engineering Manager for discrete-event simulation at MathWorks in Natick, MA. He has a Ph.D. degree in Electrical Engineering from Oklahoma State University in Stillwater, OK, and a Ph.D. degree in Control Systems from Universidad Nacional del Sur in Buenos Ares, Argentina. His email address is Omar.Orqueda@mathworks.com

DOUG EASTMAN holds an M.S. from MIT and a B.S. from UC Berkeley, both in Mechanical Engineering. He specializes in Control Systems and did thesis work in Materials Science. He is currently an Application Engineer at MathWorks. His email address is Doug.Eastman@mathworks.com. 